\title{
BEAM DYNAMICS STUDIES FOR THE SPARC PROJECT
}

\author{
M. Biagini, M. Boscolo, M. Ferrario, V. Fusco, S. Guiducci, M. Migliorati, Vaccarezza, \\ INFN-LNF, Frascati, ITALY \\ L. Serafini, INFN-Mi, Milan, ITALY \\ R. Bartolini, L. Giannessi, M. Quattromini, C. Ronsivalle, ENEA-Frascati, ITALY \\ C. Limborg, SLAC, Stanford, USA, J. B. Rosenzweig, UCLA, Los Angeles, USA
}

\begin{abstract}
The aim of the SPARC project, is to promote an R\&D activity oriented to the development of a high brightness photoinjector to drive SASE-FEL experiments. We discuss in this paper the status of the beam dynamics simulation activities.
\end{abstract}

\section{INTRODUCTION}

The SPARC photoinjector has to drive a SASE FEL experiment at $530 \mathrm{~nm}$ [1]. To meet the FEL requirements a high brightness electron beam has to be generated, accelerated up to $155 \mathrm{MeV}$ and transported up to the entrance of the undulator, minimizing the emittance and energy spread degradation due to correlated space charge and wake field effects. In order to saturate the FEL radiation in the planned $15 \mathrm{~m}$ long undulator, and to additionally allow generation of higher harmonics, the design beam parameters are very rigorous: normalized emittance $\varepsilon_{n}<1 \mu \mathrm{m}$, relative energy spread $\Delta \gamma / \gamma<0.1$ $\%$ and peak current $I \sim 90$ A. Fortunately, such parameters have to be reached only on the scale of the FEL cooperation length, which in our case is less than $300 \mu \mathrm{m}$.

\section{START TO END SIMULATIONS}

The accelerator consists of a 1.6 cell RF gun operated at S-band with a peak field on the cathode of $120 \mathrm{MV} / \mathrm{m}$ and an incorporated metallic photo-cathode followed by an emittance compensating solenoid and three accelerating sections of the SLAC type (S-band, travelling wave). A transfer line made with two triplets allows the matching with the undulator optics (see Fig. 1). A start-to-end simulation of the beam dynamics from the injector through transfer line and undulator system has been performed by means of the codes PARMELA [2] and GENESIS [3].

We take as our example the most conservative system that is to be encountered, one with no velocity bunching [1], and a relatively low energy of $155 \mathrm{MeV}$ (consistent with either low gradient, three TW section operation, or high gradient, two TW section operation). The temporal profile of the bunch has been taken uniform over $11.5 \mathrm{ps}$ with a rising time of $1 \mathrm{ps}$, a laser spot on the cathode of 1 $\mathrm{mm}$ and a $1 \mathrm{nC}$ charge, with a 35 degree launch phase in the gun, $0.27 \mathrm{~T}$ of solenoid field and on-crest acceleration in the linac. We decided to place a set of coils around the first accelerating structure $(700 \mathrm{G})$, to provide additional flexibility in the choice of the accelerating gradient (25 $\mathrm{MV} / \mathrm{m}$ in the simulations).

The transverse emittance compensation process is visible in figure 2: the emittance reaches an absolute maximum in the centre of the solenoid and it is reduced to a minimum in the drifting section, then begins again to increase. The booster entrance is located at the envelope laminar waist corresponding to an emittance relative maximum [4]. The emittance oscillation is driven by a properly matched accelerating field [5] down to an absolute minimum $(\sim 0.6 \mu \mathrm{m})$ at the linac exit where the average bunch energy is $155 \mathrm{MeV}$, high enough to damp space charge forces. The estimated thermal emittance $(0.3$ $\mu \mathrm{m})$, included in the simulation, results to be in the present design the main contribution to the total emittance.

As expected, the emittance along the transfer line and in the undulator is not anymore affected by space charge effects even when the beam has to be focused to a very small spot $(\sim 55 \mu \mathrm{m})$ to meet the undulator matching conditions.

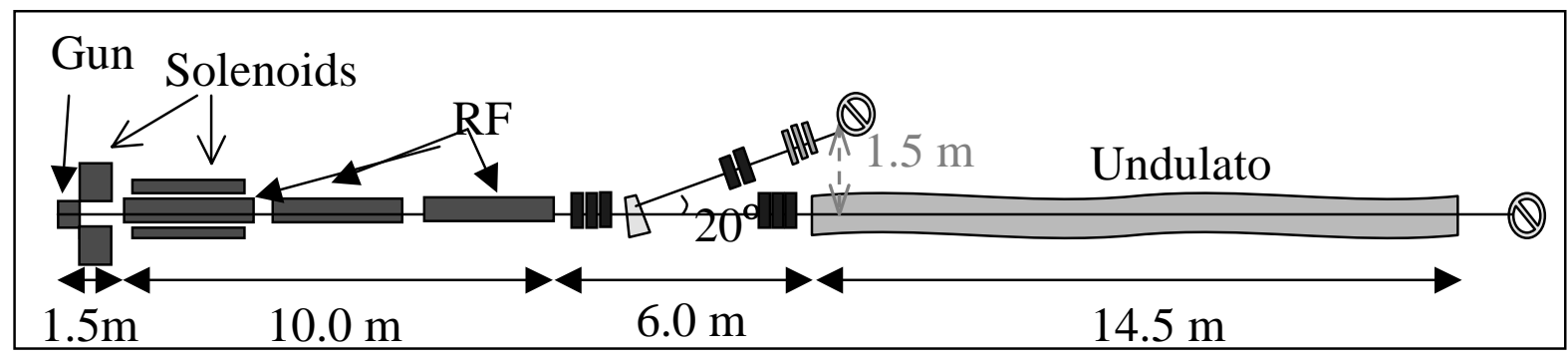

Figure 1: Schematic layout of SPARC phase 1

Transverse and longitudinal phase spaces at the entrance of the undulator are shown in figure 3 .
Despite some halo observed in the transverse phase space related to the bunch tails mismatch, the core of 
the bunch is very good behaved, having a $0.6 \mu \mathrm{m}$ rms normalized emittance in both planes.

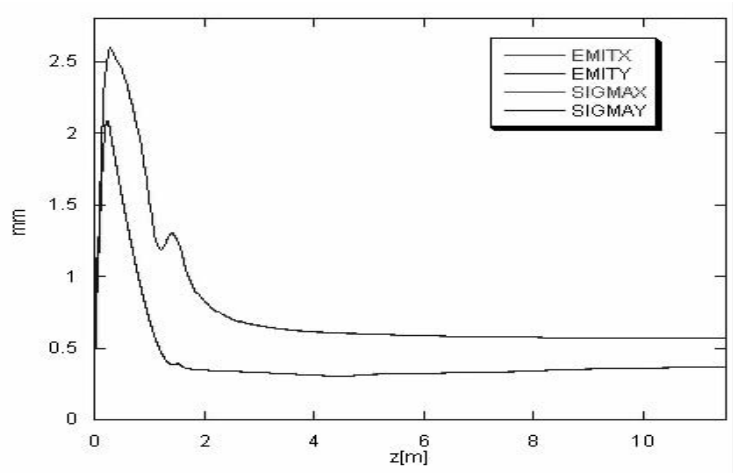

Figure 2: PARMELA simulation of the rms normalized emittance and bunch envelope evolution along the SPARC injector up to $11.5 \mathrm{~m}$.
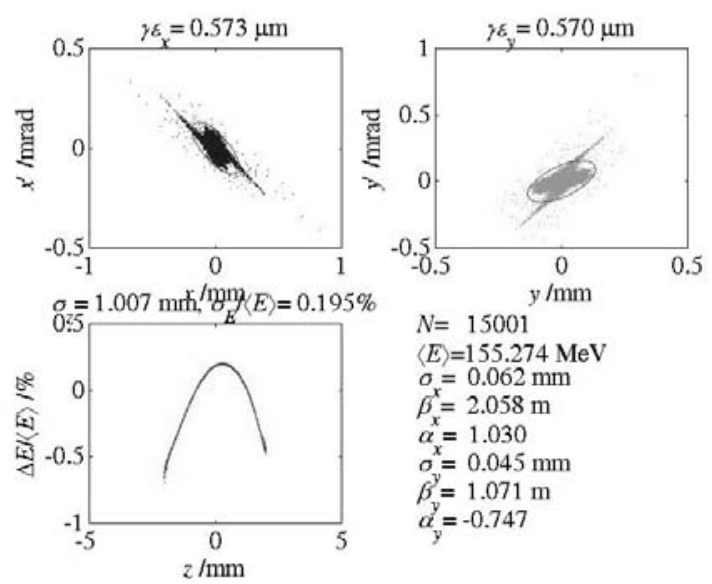

Figure 3: Transverse and longitudinal phase spaces at the entrance of the undulator.
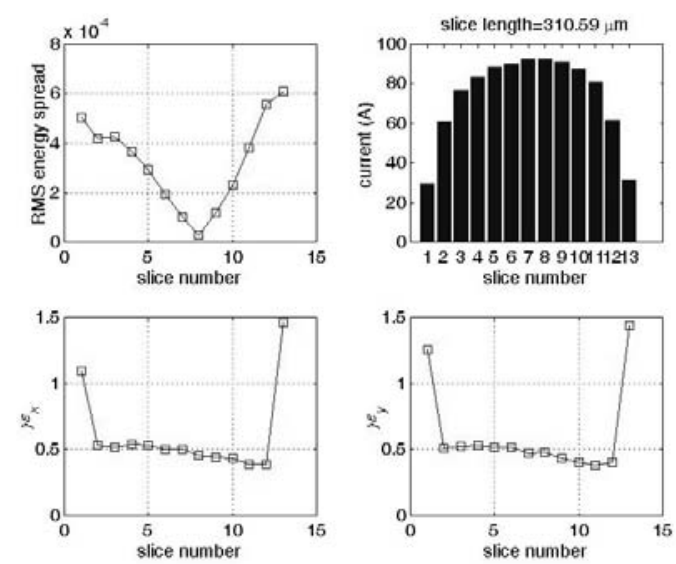

Figure 4: Slice analysis of beam properties at the undulator entrance.

The longitudinal phase space shows the typical energy phase correlation induced mainly by the RF field, with little contribution from longitudinal space-charge, having relative rms energy spread lower than $0.2 \%$ as required. The slice analysis performed at the undulator entrance is shown in figure 4. Slice emittance and energy spread are well below the nominal design values for more than $85 \%$ of the bunch length. The slice peak current is above $60 \mathrm{~A}$ in the same region.

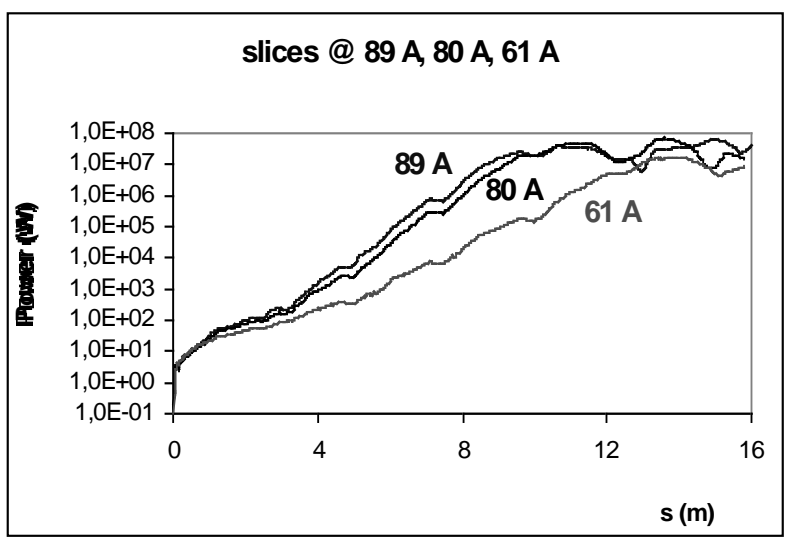

Figure 5: GENESIS simulations of the FEL for three representative slices along the bunch.

The analysis of the FEL performance has been performed with the code GENESIS, taking in to account three representative slices along the bunch provided by PARMELA, showing that even the radiation emitted by the slices closer to the tails that contain a lower local value of the current $(61 \mathrm{~A})$ can reach saturation as shown in figure 5 .

\section{SENSITIVITY STUDIES}

Once the nominal parameters are set we have to provide also stable operation and tolerate some jitter in the nominal value of the parameters. In this section we discuss only two selected topics of such studies performed thus far, in which cathode emission uniformity, laser beam ellipticity, laser centroid offset, laser time structure, and solenoid field errors have been explored.

The effects of cathode's dishomogeneities [6] have been tested by simulating, using the code TREDI [7], a zone on the cathode with reduced quantum efficiency QE. We have assumed a circular QE "hole" with a surface $10 \%$ of the nominal bunch spot, centered halfway of the bunch radius. The results suggest that localized inhomogeneities do not dramatically degrade the emittance, growing by $\Delta \varepsilon_{x(y)} \approx 10 \%(15 \%)$ for $\mathrm{QE}$ $\approx 70 \%$ as reported in figure 6 . This analysis will be further extended to the cases of randomly distributed ("spotty") inhomogeneities, which better describe the behavior of real cathodes.

Flat top laser pulses with rise times shorter than $1 \mathrm{ps}$ are required in order to avoid emittance degradation [8], as shown in figure 7. 


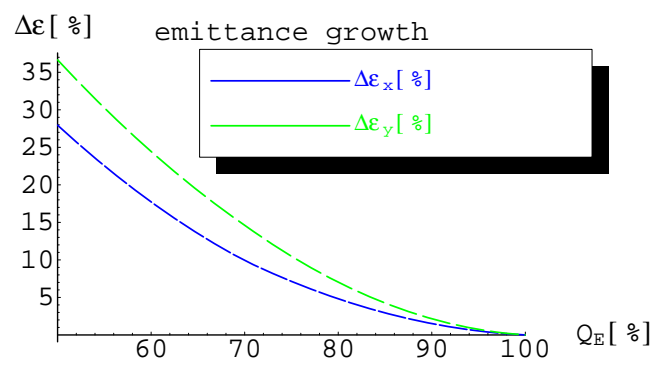

Figure 6: Emittance growth as a function of $Q_{E}$.

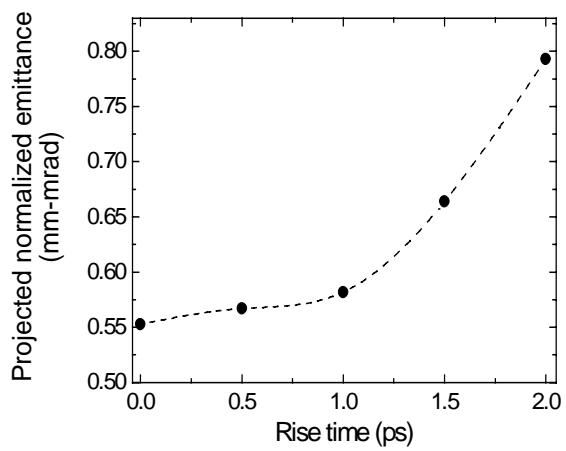

Figure 7: Norm. rms emittance versus rise time.

But laser pulse shaping with short rise time may result in the formation of longitudinal ripples. Therefore, the effect of a laser pulse with longitudinal ripples has been investigated. The beam is assumed to be transversely uniform in these studies. The striking result is that even with a 30\% of longitudinal irregularity the beam emittance degradation is limited to $6 \%$. This result can be interpreted as if the spacecharge force induces a compensation of the longitudinal irregularity. This hypothesis is justifed by PARMELA simulation results. In figure 8 the evolution of longitudinal beam distributions are displayed. At the cathode the temporal spectrum has a $30 \%$ ripple overlapped on a square pulse, the relative energy spread is zero. As the beam goes through the gun and drifts the temporal oscillations transform in energy oscillations. At the entrance of the first acceleration structure (at $z=150 \mathrm{~cm}$ ) the beam has lost the temporal ripples, which have converted into energy variations through a fractional plasma oscillation. These energy ripples do not have any notable effect to the rms energy spread at the end of the linac, as they are soon suppressed inside the first accelerating structure. These results indicate that the shape of the laser pulse should be square with a very small rise time, whether a smooth temporal profile is not a stringent requirement.

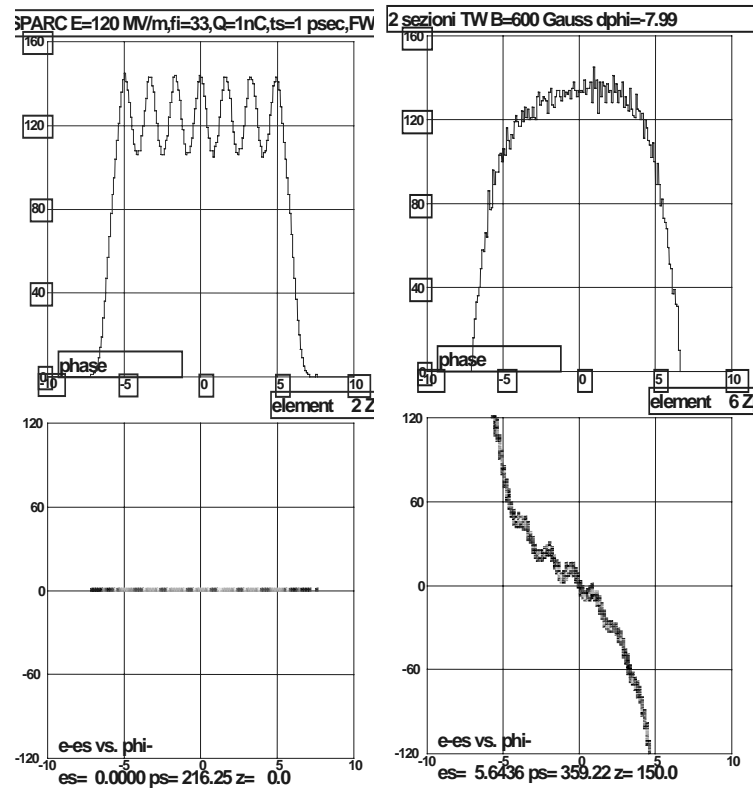

Figure 8: Longitudinal beam distribution: phase and energy spectrum in the upper and lower plots, respectively. Left plot: initial distribution at cathode $(\mathrm{z}=0 \mathrm{~m})$; right plot: beam at $\mathrm{z}=1.5 \mathrm{~m}$.

\section{CONCLUSIONS}

Start to end simulation of the optimal SPARC working point have shown that we can meet the FEL requirements with a reasonable parameter set. The requirements on the longitudinal and transverse profile of the laser pulse, the phase jitters, the laser pointing stability have been set. Preliminary sensitivity studies have not shown dramatic concerns about the possibility of stable operation. A more systematic [9] study is in progress .

\section{REFERENCES}

[1] L. Palumbo et al., "The SPARC Project: A HighBrightness Electron Beam Source to Drive a SASEFEL Experiment at LNF", this conference.

[2] J. Billen, "PARMELA", LA-|UR-96-1835, 1996.

[3] S. Reiche, Nucl. Instrum. \& Meth. A429,243 (1999).

[4] M.Ferrario et al., "HOMDYN Studies for the LCLS RF Photoinjector", Proc. of ICFA Workshop on the Physics of High Brightness Beams, UCLA, Nov. 1999

[5] L.Serafini,J.Rosenzweig,Phys. Rev. E 55(1997) 7565

[6] F. Zhou et al.,PRST-AB, V. 5, 094203 (2002).

[7] L. Giannessi, M. Quattromini, Submitted to PRST AB (2003).

[8] J. Yang, J. of Appl. Phys., V. 92, N. 1, (2002)

[9] C. Limborg, "New optimization for the LCLS photo-injector", Proc. Of EPAC-02, Paris 2002. 